ORIGINAL ARTICLE

\title{
The diversity of the opa gene in gonococcal isolates from men who have sex with men
}

\author{
F Howie, H Young, A McMillan
}

Sex Transm Infect 2004;80:286-288. doi: 10.1136/sti.2003.006775

See end of article for authors' affiliations

a.r.

Correspondence to: Dr H Young, Scottish Neisseria gonorrhoeae Reference Laboratory, Department of Laboratory Medicine, Edinburgh Royal Infirmary, 51 Little France Crescent, Edinburgh EH16 4SA, UK; Hugh.Young@ luht.scot.nhs.uk

Accepted for publication 3 November 2003 Objectives: To use a molecular typing method (opa-typing) to characterise gonococcal isolates obtained
from men who have sex with men (MSM) attending a genitourinary medicine (GUM) clinic in Edinburgh during 2001. To compare the results of opa-typing with A/S (auxotype/serovar) phenotyping, and with epidemiological information obtained by contact tracing.

Methods: Isolates were opa-typed by a PCR-RFLP method where the restriction fragments resulting from digestion with three separate restriction enzymes were resolved by polyacrylamide gel electrophoresis. Isolates were considered to have the same opa-type if the band patterns were identical or if they differed by one band between the three digestions.

Results: 40 opa-types were detected from a collection of 73 gonococcal isolates from 61 patients. 26 opatypes were unique, being found in one individual each, 14 different opa-types were found in more than one patient. Opa-typing was found to have a discrimination index (DI) of 0.96, compared to a DI of 0.87 for A/S phenotyping, indicating that opa-typing is better at discriminating between unrelated isolates. Opa-typing confirmed three epidemiological links established by contact tracing, and uncovered a further 13 clusters of isolates.

Conclusions: Opa-typing is a more discriminative method than $\mathrm{A} / \mathrm{S}$ phenotyping when determining relatedness in gonococcal isolates. The ability of opa-typing to identify sexual networks not disclosed by contact tracing and conventional phenotyping make it a useful method for studying the spread of gonorrhoea with the potential to contribute to the control of this infection.

G onorrhoea is a major sexually transmitted infection that contributes a significant burden on the resources of health services. Despite treatment being available, gonococcal infection remains poorly controlled especially in high risk groups such as, men who have sex with men (MSM). ${ }^{1}$

Typing of gonococcal isolates can potentially enhance the control of gonococcal infection, and in conjunction with social and epidemiological data can theoretically provide valuable information on the spread of gonorrhoea within a community. ${ }^{2}$ Phenotypic typing based on the A/S (auxotyping/serotyping) system is commonly used across the United Kingdom; however this typing system is unable to detect short term transmission chains and is open to errors in interpretation. ${ }^{3}$

Techniques such as opa-typing may be particularly useful in addressing the detection of short term transmission. ${ }^{4} \mathrm{~A}$ single gonococcus possesses up to 11 copies of the opa gene, ${ }^{56}$ which are scattered throughout the gonococcal genome and are subject to genetic recombination. The extensive variation and rapid evolution of the opa gene repertoire suggests that isolates should have different opa-types unless they are part of a recently linked transmission network. ${ }^{4}$ It is theoretically possible to confirm a link between two patients or even detect links between patients unsuspected of known contact. ${ }^{7}$

Opa-typing may be especially useful in profiling gonococcal isolates from men who have sex with men (MSM). As contact tracing in the MSM group is difficult molecular typing may be useful in defining existing sexual networks not recognised by conventional phenotyping or contact tracing methods. ${ }^{7}$ A previous study ${ }^{8}$ showed that opa-typing was able to describe potential sexual networks but this was more successful in smaller closed communities. The aims of this study were to opa-type the gonococcal isolates from MSM who attended a GUM clinic over a 1 year period; compare the discriminatory power of opa-typing and conventional phenotyping; compare transmission links suggested by opatyping with epidemiological information obtained by contact tracing.

\section{MATERIALS AND METHODS}

Clinical isolates used in this study represented 94\% (62/66) of all MSM with culture proved gonorrhoea attending a GUM clinic in Edinburgh during the year 2001. Isolates were inoculated onto modified New York City agar plates (Biomerieux, UK) and thick suspensions of bacterial growth made in $1 \mathrm{ml}$ of sterile distilled water. The suspensions were boiled for 10 minutes, centrifuged for 5 minutes at $1300 \mathrm{rpm}$ and the supernatants transferred to fresh microcentrifuge tubes for storage at $-20^{\circ} \mathrm{C}$ until required.

The opa genes were amplified using opa up, forward, and reverse primers (fwd-GCC ATT ATT TCA GAA ACA TCC and rev-GCT TCG TGG GTT TTG AAG CG) according to the simplified opa-typing method used by Palmer et al. ${ }^{9}$

The presence of polymerase chain reaction (PCR) products was determined using agarose gel electrophoresis ( $1 \%$ agarose gel in tris-acetate-EDTA buffer). Desired DNA bands were approximately $550 \mathrm{bp}$.

PCR products were purified using QIAquick spin columns (Qiagen, UK) before digestion with Taq 1, Hhal, or Alul restriction enzymes. After the restriction reaction, the products were separated by polyacrylamide gel electrophoresis (PAGE) using a non-denaturing $12 \%$ polyacrylamide gel in TAE buffer (Biorad, UK). Gels were stained in ethidium bromide and comparison of bands was done visually using ultraviolet light.

Abbreviations: DI, discrimination index; HAs, health advisers; MSM, men who have sex with men; PAGE, polyacrylamide gel electrophoresis; PCR-RFLP, polymerase chain reaction-restriction fragment length protein 
Isolates were considered to have the same opa-type if the band patterns were identical or if they differed by one band between the three digestions. Each distinct restriction pattern was given an arbitrary opa designation.

\section{Correlation with contact tracing and phenotyping}

Partner notification was undertaken by trained health advisers (HAs). The index patient (and secondary contacts) was interviewed by the HA, and details of the sexual contact, including name if known, were noted. Either the patient or HA contacted the named individual who was invited to attend the clinic for investigation and treatment. The HA linked the sexual contacts to the index case. As ethics approval was for an unlinked anonymous study no demographic data were available: once the contact information had been provided all case numbers and isolates were given separate study numbers and the case number removed from the database. Duplicate isolates from the same case remained linked and we also knew that patient A was a contact of patient $\mathrm{D}$ but not the case number for these patients.

The A/S phenotypes of the isolates used in this study had been determined before cold storage as part of the Scottish Neisseria gonorrhoeae Reference Laboratory service. ${ }^{1011}$ Simpson's index of diversity ${ }^{12}$ was used to assess the discrimination of the A/S phenotyping and the opa-typing techniques.

\section{RESULTS}

A total of 73 isolates were recovered from 62 of the 66 patients; 51 of the patients yielded gonococcal isolates from a single anatomical site, and 11 yielded isolates from two anatomical sites. The 73 isolates were subdivided into four auxotypes, 13 serovars, $18 \mathrm{~A} / \mathrm{S}$ classes, and 40 opa-types.

Simpson's index of diversity gave a discrimination index of 0.39 for auxotyping, 0.83 for serotyping, 0.87 for the $\mathrm{A} / \mathrm{S}$ combination, and 0.96 for opa-typing. Table 1 shows the distribution of isolates within the various A/S classes and the number of opa-types associated with each A/S class while figure 1 shows the distribution and relation of the 40 opatypes for the population of isolates.

Of the 40 opa-types, 26 (65\%) were unique and 14 (35\%) formed clusters (see fig 1). Of the unique opa-types there were 19 individuals with infection at a single anatomical site and six with infection at two anatomical sites; of these six, four patients had infection with unique opa-types and two patients were infected with distinct opa-types, which differed by several bands, at each anatomical site. The 14 opa clusters comprised six clusters with pairs of isolates, three with three isolates, four with four isolates, and one of six isolates.

Contact tracing revealed seven pairs with known sexual contact; three contact pairs had concordant opa-types while gonococcal cultures were negative in four patients. Opatyping identified a further 11 indistinguishable pairs or clusters.

\section{DISCUSSION}

Our results demonstrate that the increased discrimination achieved by opa-typing identified links between isolates that would not have been detected by conventional methods. Because opa-typing can discriminate within A/S classes it is potentially useful in defining sexual networks within populations. It is impossible to define sexual networks with $\mathrm{A} / \mathrm{S}$ phenotyping as a few $\mathrm{A} / \mathrm{S}$ classes predominate in the population. The definition of sexual networks within a population as an aid to understanding and controlling gonococcal infection within a locality is also limited if a very large proportion of isolates belong to the same serovar.

A previous opa-typing study involving populations from London and Sheffield found that $62 \%$ of isolates from London and $21 \%$ from Sheffield had unique opa-types. ${ }^{78}$ London had no large clusters while Sheffield had two large clusters with 18 and 43 cases in each. Our finding of 37\% of isolates belonging to unique opa-types lies between the Sheffield and London figures. However, it is difficult to compare the results of these studies directly because our population is restricted to MSM, whereas the individuals in the other studies included heterosexuals. ${ }^{78}$ As far as we are aware, this is the first study where opa-typing has been used in a population of MSM.

Routine contact tracing identified three linked clusters, while opa-typing identified 14 clusters. Although all epidemiological links via contact tracing were confirmed using the opa-typing method in our study, in other studies discordance was found in some contact pairs. ${ }^{78}$ These discrepancies do not necessarily imply other sexual partners. Discordance could be the result of natural evolution of the gene or selection of two different opa-types that may be present in the patient. ${ }^{8}$

Opa-typing was more useful than phenotyping, in identifying individuals infected with more than one gonococcal strain. Of the two individuals who had two different opatypes, one had isolates which also differed in both auxotype and serotype, while the other was infected with two isolates of the same A/S class.

Table $1 \mathrm{~A} / \mathrm{S}$ class of 73 gonococcal isolates and number of different opa-types within each $\mathrm{A} / \mathrm{S}$ class

\begin{tabular}{llll}
\hline Serotype & Auxotype & No in each class & No of opa-types within class \\
\hline Brpyust & NR & 16 & 12 \\
Bropyst & NR & 18 & 10 \\
Brpyut & P & 5 & 2 \\
Bpyust & A & 6 & 1 \\
Bropt & NR & 5 & 2 \\
Bpyvut & NR & 5 & 2 \\
Bpyst & NR & 4 & 2 \\
Broput & NR & 2 & 2 \\
Broput & PA & 2 & 1 \\
Brpyu & P & 2 & 1 \\
Bropst & NR & 1 & 1 \\
Brpyut & NR & 1 & 1 \\
Brpyut & A & 1 & 1 \\
Bpyust & P & 1 & 1 \\
Bopyst & NR & 1 & 1 \\
Bpyt & NR & 1 & 1 \\
Arost & P & 1 & 1 \\
Bpyust & NR & 1 & \\
\hline
\end{tabular}




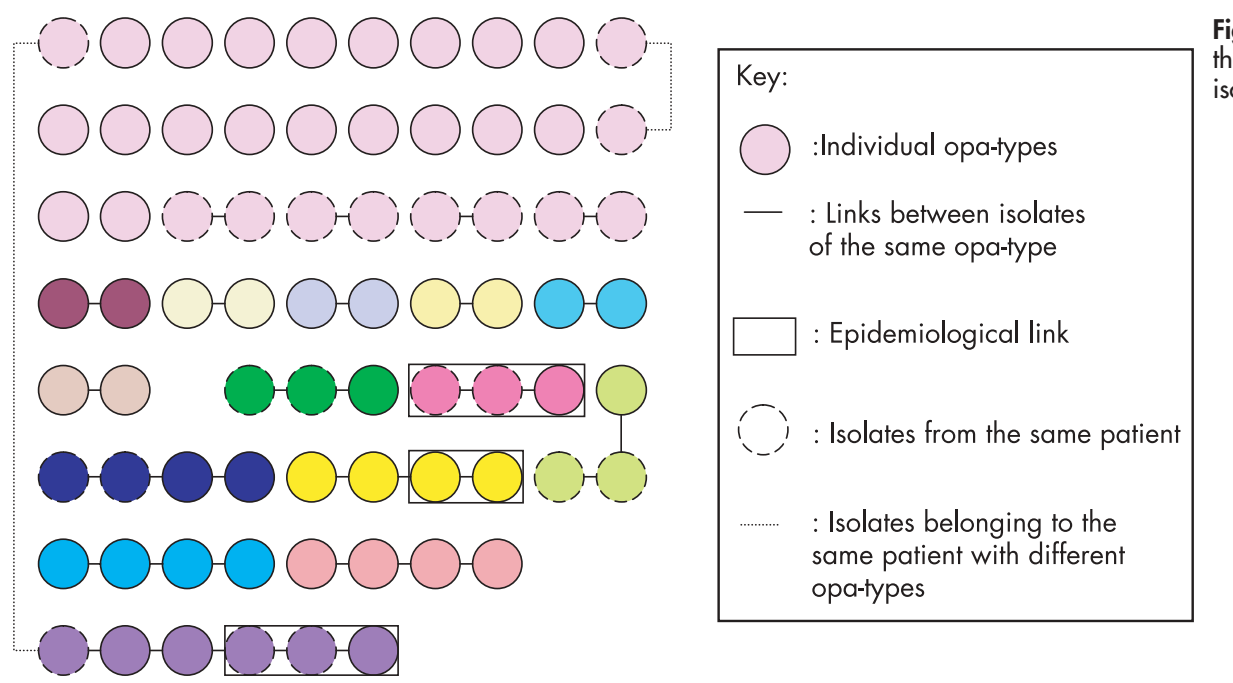

Figure 1 Distribution and relation of the 40 opa-types for the population of isolates.

\section{Key messages}

- Opa-typing is more discriminatory than phenotyping using a combination of auxotype and serovar

- Opa-typing can identify links between isolates not established by conventional methods

- Opa-typing combined with patient interview has the potential to guide appropriately targeted preventative interventions

- Molecular typing methods such as opa-typing may be particularly valuable in increasing our understanding of gonococcal transmission in MSM.

In conclusion, opa-typing offers greater discrimination than $\mathrm{A} / \mathrm{S}$ phenotyping when examining a population of MSM. Opa-typing confirms epidemiological links ascertained by contact tracing, and identified potential links in the absence of any contact information, and has the potential to contribute to the control of gonorrhoea through improved understanding of transmission within a population such as MSM. The community of MSM in Edinburgh is fairly diverse. The city is a popular tourist destination for MSM from all over the world, and sexual contacts may be made in one of four or five bars or clubs, and in "cruising" areas. Opa-typing combined with patient interview could highlight specific locations where high levels of transmission occur or highly resistant strains introduced thus allowing appropriately targeted interventions.

\section{ACKNOWLEDGEMENTS}

We thank Dr Helen Palmer for help in interpreting opa-typing results.

\section{CONTRIBUTORS}

FH undertook this study as part of an MSc in molecular medical microbiology at the Department of Laboratory Sciences, University of
Nottingham; FH was responsible for the opa-typing and drafting the manuscript; HY initiated the project, provided supervision throughout, and contributed to the writing of the final manuscript; AMcM contributed to the design of the study, provided epidemiological information, and contributed to the writing of the final manuscript.

\section{Authors' affiliations}

F Howie, H Young, Scottish Neisseria gonorrhoeae Reference Laboratory, Department of Laboratory Medicine, Edinburgh Royal Infirmary, 51 Little France Crescent, Edinburgh EH16 4SA, UK

A McMillan, Department of Genitourinary Medicine, Lothian University Hospitals NHS Trust, Lauriston Building, Lauriston Place, Edinburgh, UK

Ethical approval for this project was given by the Lothian Research Ethics Committee (LREC 2002/8/32).

\section{REFERENCES}

1 Van Duynhoven YTHP. The epidemiology of Neisseria gonorrhoeae in Europe. Microbes and Infection 1999;1:455-64.

2 Van Looveren $M$, Ison $C A$, leven $M$, et al. Evaluation of the discriminatory power of typing methods for Neisseria gonorrhoeae. J Clin Microbiol 1999; 37:2183-8.

3 Ison CA. Genotyping of Neisseria gonorrhoeae. Curr Opin Infect Dis 1998;11:43-6.

4 O'Rourke M, Ison CA, Renton AM, et al. Opa-typing: a high resolution tool for studying the epidemiology of gonorrhoea. Mol Microbiol 1995; 17:865-75.

5 Meyer TF. Pathogenic Neisseriae: complexity of pathogen-host cell interplay. Clin Infect Dis 1999;28:433-41.

6 Dehlio C, Gray-Owen SD, Meyer TF. The role of Neisserial opa proteins in interactions with host cells. Trends Microbiol 1998;6:489-95.

7 Day $\mathrm{S}, \mathrm{Ward} \mathrm{H}$, Ison $\mathrm{C}$, et al. Sexual networks: the integration of social and genetic data. Soc Sci Med 1998:47:1981-92.

8 Ward $\mathrm{H}$, Ison CA, Day SE, et al. A prospective social and molecular investigation of gonococcal transmission. Lancet 2000;356:1812-17.

9 Palmer HM, Leeming JP, Turner A. Investigation of an outbreak of ciprofloxacin resistant Neisseria gonorrhoeae using a simplified opa-typing method. Epidemiol Infect 2001;126:219-24.

10 Copley CG, Egglestone SI. Auxotyping of Neisseria gonorrhoeae isolated in the United Kingdom. J Med Microbiol 1983;16:295-302.

11 Moyes A, Young H. Epidemiological typing of Neisseria gonorrhoeae: a comparative analysis of three monoclonal antibody serotyping panels. Eur J Epidemiol 1991;7:311-19.

12 Dillon JR, Rahman M, Yeung K-H. Discriminatory power of typing schemes based on Simpson's index of diversity for Neisseria gonorrhoeae. J Clin Microbiol 1993;31:2831-3. 